\title{
Lymphoma of the Nasopharynx in an HIV Sero-Positive Patient in the General Hospital of Douala
}

\author{
Gisele Alenda Ngulefac, Jean Valentin Fokouo Fogha, Esthelle Genevieve Stephanie Minka Ngom, \\ Vincent Fonyam, Louis Richard Njock
}

ENT-HNS, General Hospital of Douala, Douala, Cameroon.

Email: ngualenda@yahoo.com

Received December $11^{\text {th }}, 2011$; revised January $18^{\text {th }}$, 2012; accepted February $14^{\text {th }}, 2012$

\begin{abstract}
Humman immunodeficiency virus (HIV) infection is associated with opportunistic cancers notably Non-Hodgkin lymphoma (NHL). Generally the response to chemotherapy or radiation therapy is satisfactory but requires nursing measures to protect these patients from superinfection to which they are highly predisposed by the double immunosuppression. We report the case of a 54 years-old known HIV sero-positive female with lymphoma of the nasopharynx who presented lately and died of septic shock despite the favorable evolution of the tumor on treatment.
\end{abstract}

Keywords: HIV Infection; Non-Hodgkin Lymphoma; Double Immunosuppression

\section{Introduction}

Cancers of the nasopharynx represent the first cause of cancers of the ENT sphere in Cameroon. The histology is dominated by non-differentiated carcinomas (Undifferentiated carcinoma of nasopharyngeal type-UCNT) [1]. NonHodgkin lymphomas represent up to $4 \%$ of cancers and usually affect the head and neck [2]. With the HIV/ AIDS pandemic, there is an up rise in the frequency of NHL [3], which with lymphomas of the central nervous system are AIDS defining conditions [3]. We describe the case of NHL of the nasopharynx in an HIV seropositive patient with significant regional extension.

\section{Clinical Case}

It was the case of a 54 year-old HIV sero-positive female on ARV since 6 years (Lamivudine-Zidovudine-Efavirenz). She was brought by her family members for an altered general state with asthenia and weight loss of about $10 \%$ initial body weight. She also suffered from dysphagia to solids since 6 months, a right lateral cervical tumefaction since 8 months of increasing size causing dyspnoea. A biopsy was done in the $4^{\text {th }}$ month of evolution but the sample was not analysed. The patient was placed on anti-tuberculosis quadritherapy without amelioration and this motivated the stop of treatment after 2 months. She thereafter underwent traditional scarifications.

On admission we noted

- A right lateral cervical multinodular mass extending from the submandibular region to the right supraclavicular region as shown in Figure 1. It measured about
$12 \mathrm{~cm}$ in main axis with superficial ulcerations and discharge. There was significant infiltration. The surrounding skin had pitting oedema.

- A superior cava syndrome with voluminous oedema of the head giving a moon face and also of the right upper limb.

- Bilateral nasal obstruction with snoring, buccal respiration and hyponasality and a budding non-ulcerated mass of the right Rosenmuller fossa on nasal endoscopy.

- There was no sign of respiratory distress.

- Her CD4 count was $10 / \mathrm{mm}^{3}$, the CD3 count was $616 / \mathrm{mm}^{3}$ and the CD8 count was $603 / \mathrm{mm}^{3}$. This classified the patient as C3 of the CDC of Atlanta system. Initial treatment consisted of antibiotherapy with Ceftazidime and Metronidazole, bi-daily dressing with Dakin Cooper solution and continuation of her ARV.

A fine needle aspirate of the cervical mass revealed a diffuse centroblastic NHL. A biopsy of the nasopharynx confirmed the diagnosis of NHL. Workup of extension of disease revealed that the other lymph node areas were free as well as other lymphoid organs.

She was placed on chemotherapy following a multidisciplinary therapeutic decision-making meeting with ENT, medical oncologist, radiation oncologist and internist. The protocol used was CHOP-Bleo (Cyclophosphamide, Adriablastine, Vincristine, Cortancyl and Bleomycine). After the first cure, the cervical mass regressed by about $70 \%$ of its initial size and there was regression of all rhinologic and pharyngeal symptoms. Figure 2 show relative decrease in swelling of neck following first chemotherapy 


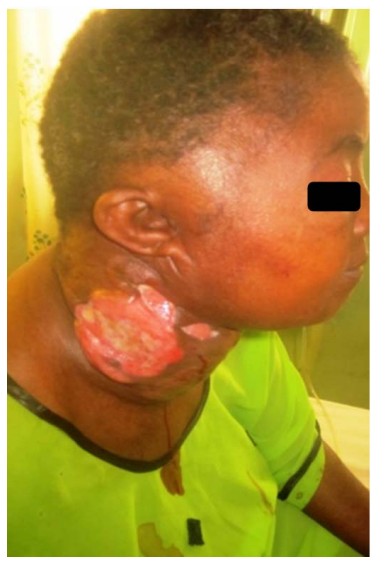

(a)

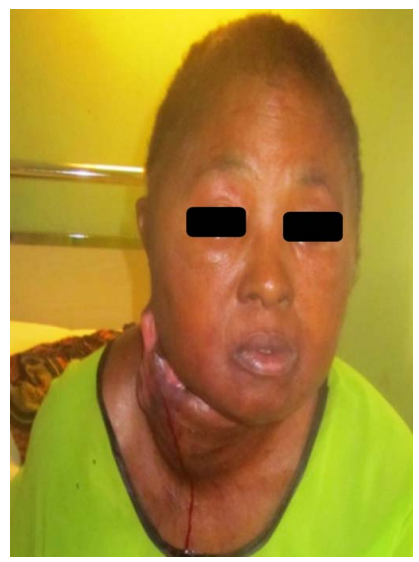

(b)
Figure 1. Initial clinical aspect.

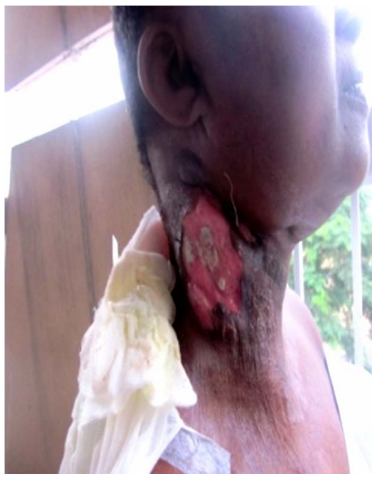

(a)

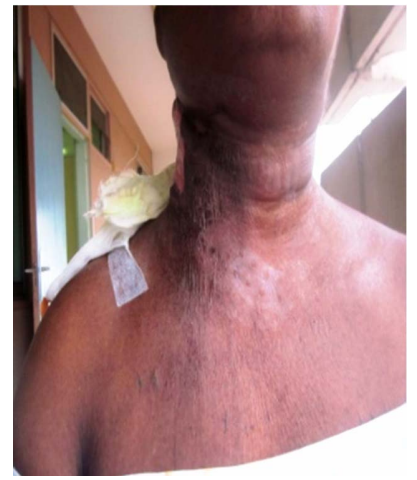

(b)
Figure 2. After first session of chemotherapy.

cure.

Evolution was however deceptive as the patient developed a septic shock on severe neutropenia.

\section{Discussion}

NHL affects the head and neck frequently, with both nodal and extra-nodal locations. Even though not directly implicated in the management, the ENT specialist is at the centre of diagnosis.

After 8 months of inappropriate management of this patient, the diagnosis of NHL was made. Late diagnosis is an usual phenomenon noticed by Yomi [4] and Batueca Caletrio [2]. This lateness is explained by the distancing of the patient who came from a remote area of the country and by their belief in the efficacy of traditional treatment.

The age of our patient, 54 years, falls in the interval of 40 to 80 years which is reported by most authors [3,5]. The clinical presentation is similar to that reported in li- terature, where there is predominance of lymphadenopathies [2], followed by pharyngeal obstructive manifestations (nasal obstruction, dysphagia, snoring). The contrast between the voluminous lymphadenopathies and the small primitive tumour was noticeable. This lymphophilia of cancers of the nasopharynx is reported by many authors $[2,6]$. The superior cava syndrome is unusual with these tumours. We did not find other reported cases.

Histology revealed a diffuse centroblastic NHL. The absence of immune-histo-chemistry did not permit to precise the subtype B or T. But literature reports a marked predominance of type b lymphomas $[2,3]$.

The high sensitivity of nasopharyngeal lymphoma to chemotherapy is known [3]. But the absence of appropriate structures for management of patients with double immunosuppression led to the death of this patient, following an expected complication of chemotherapy.

\section{Conclusion}

The relationship between NHL and AIDS is known. This case permits for us to bring out the notion that patients with association of immunosuppression and lymphoma can have a poor prognosis in our context of management with a limited technical platform whereas this association of HIV/AIDS and lymphoma is not rare in our environment.

\section{REFERENCES}

[1] S. A. Mouelle, A. Fouda and P. Ndom, "Aspects Evolutifs des Cancers du Cavum Traités à l'Hôpital Général de Douala," Médecine d'Afrique Noire, Vol. 48, No. 3, 2001, pp. 97-101.

[2] C. A. Batuecas, H. A. Muñoz, P. P. Blanco, J. M. S. López and M. M. Gil, "Non Hodgkin's Lymphoma in the ENT Field,” Acta Otorrinolaringológica Española, Vol. 56, No. 5, 2005, pp. 215-218.

[3] M. Corti, M. F. Villafane, M. Cermelj, et al., "Cavum Lymphoma in a Hemophilic Patient with AIDS," Medicina (B Aires), Vol. 60, No. 3, 2000, pp. 351-353.

[4] J. Yomi, E. Fongang and A. G. Juimo, "The Value of Medical Imaging in the Diagnosis and Assessment of Local and Regional Extension of Cancers of the Cavum at Yaounde,” Sante, Vol. 9, No. 4, 1999, pp. 231-234.

[5] A. S. A. Arfaoui, A. Quyou, F. Habib and C. M. Khaled, "Le Cancer du Cavum au Maroc. Etude Epidémiologique sur l'Echantillon: Centre d'Oncologie Al Azhar de Rabat," Antropo, Vol. 14, 2007, pp. 75-82.

[6] H. B. N. Boussen, A. Gamoudi, N. Mokni, F. Benna, I. Boussen and A. Ladgham, "Cancers du Nasopharynx in Encyclopédie Médico-Chirurgicale ORL,” E. M. SAS, Paris, 2007. 